\title{
The one-carbon metabolism pathway highlights therapeutic targets for gastrointestinal cancer (Review)
}

\author{
MASAMITSU KONNO ${ }^{1}$, AYUMU ASAI ${ }^{1-3}$, KOICHI KAWAMOTO ${ }^{2}$, NAOHIRO NISHIDA ${ }^{2}$, \\ TAROH SATOH $^{1}$, YUICHIRO DOKI ${ }^{1-3}$, MASAKI MORI ${ }^{1-3}$ and HIDESHI ISHII ${ }^{1,3}$ \\ Departments of ${ }^{1}$ Frontier Science for Cancer and Chemotherapy, ${ }^{2}$ Gastroenterological Surgery Graduate \\ School of Medicine, and ${ }^{3}$ Cancer Profiling Discovery, Osaka University, Osaka 565-0871, Japan
}

Received November 17, 2016; Accepted December 22, 2016

DOI: 10.3892/ijo.2017.3885

\begin{abstract}
After the initial use of anti-folates for treatment of malignancies, folate metabolism has emerged as a rational diagnostic and therapeutic target in gastrointestinal cancer. The one-carbon metabolic pathway, which comprises three critical reactions (i.e., folate and methionine cycles), underlies this effect in conjunction with the trans-sulfuration pathway. Understanding of the one-carbon metabolism pathway has served to unravel the link between the causes and effects of cancer phenotypes leading to several seminal discoveries such as that of diadenosine tri-phosphate hydrolase, microRNAs, 5-FU and, more recently, trifluridine. In the folate cycle, glycine and serine fuel the mitochondrial enzymes SHMT2, MTHFD2 and ALDH1L2, which play critical roles in the cancer survival and proliferation presumably through purine production. In the methionine cycle, $\mathrm{S}$-adenocyl methionine serves hydrocarbons and polyamines that are critical for the epigenetic controls. The trans-sulfuration pathway is a critical component in the synthesis of glutathione, which is involved in the production of reactive oxygen species in cancer stem cells. Therefore, characterization of one-carbon metabolism is indispensable to the development of precision medicine in the context of cancer diagnostics and therapeutics. In the present study, we review the historical issues associated with one-carbon metabolism and highlight the recent advances in cancer research.
\end{abstract}

\section{Contents}

1. The multi-faceted one-carbon metabolic pathway

2. Therapeutic targets in $\mathrm{C} 1$ metabolism

Correspondence to: Prof. Masaki Mori or Prof. Hideshi Ishii, Department of Cancer Profiling Discovery, Osaka University, Graduate School of Medicine, Suita, Yamadaoka 2-2 Osaka 565-0871, Japan

E-mail: mmori@gesurg.med.osaka-u.ac.jp

E-mail: hishii@gesurg.med.osaka-u.ac.jp

Key words: one-carbon metabolism, gastrointestinal cancer, cancer stem cells
3. Application of nucleotide analogues in $\mathrm{C} 1$ metabolism

4. Polyamines in $\mathrm{C} 1$ metabolism

5. Diadenosine phosphate hydrolases in $\mathrm{C} 1$ metabolism

6. ROS in $\mathrm{C} 1$ metabolism

7. Roles in cancer stem cell control

\section{The multi-faceted one-carbon metabolic pathway}

According to central dogma, information flow from the genome is dictated by the transcription of coding genes to mRNA, followed by translation to proteins. Multi-faceted omics information yields high-volume data associated with the whole-genome sequence, epigenome, methylome, transcriptome, proteome and metabolome, all of which have been linked to disease-specific cell phenotypes (1). The metabolome comprises of physiologically active substances such as nutrients (e.g., glucose), lipids, amino acids (e.g., serine and glycine) and nucleic acids. Importantly, in tumor cells, the processes of cell growth and proliferation requires construction of building blocks for new cellular components from substances associated with a redox status (Fig. 1) (2). One-carbon (C1) metabolism encompasses a complex metabolic network based on the chemical reaction of folate compounds (3). The folate cycle couples with the methionine cycle to form a bi-cyclic metabolic pathway that circulates carbon units as part of a process referred to as the $\mathrm{C} 1$ metabolism (3). These two cycles also link with the trans-sulfuration pathway, which plays a critical role in the regulation of the redox state by producing glutathione (3). C1 metabolism is critical for the maintenance of genomic stability through nucleotide metabolism as well as for the epigenetic control of DNA and histones, altered expression of which is a characteristic attribute of tumor cells. Ultimately, these findings should unravel new opportunities for translational approaches, drug discovery and studies of cancer pathogenesis. The study and control of $\mathrm{C} 1$ metabolism is the foundation for precision medicine in the context of disease prevention, identification of biomarkers, diagnosis, and treatment of various diseases, including cancer (3-5). High expression of $\mathrm{C} 1$ metabolic enzymes such as SHMT2, MTHFD2 and ALDH1L2 was shown to be independently associated with RFS. These findings suggest that mitochondrial folate metabolic enzymes could serve as potential therapeutic 


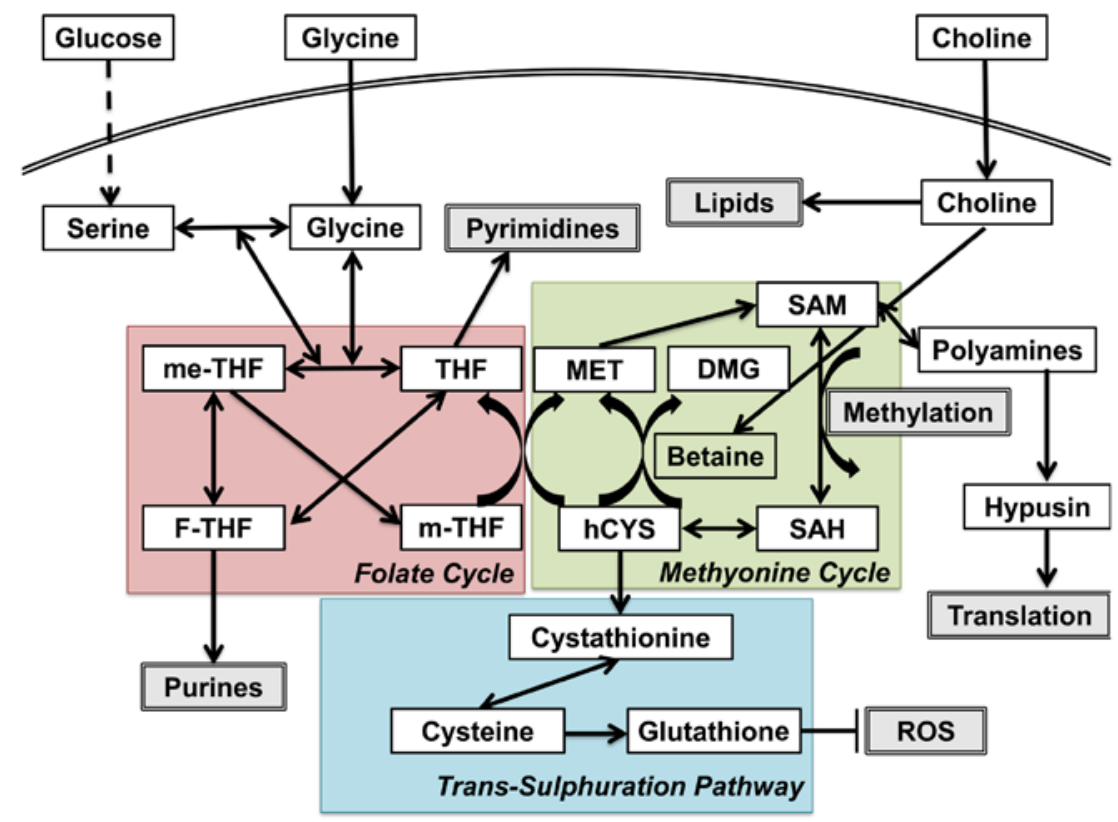

Figure 1. Multi-faceted functions of one-carbon metabolism. Three mitochondrial enzymes, SHMT2, MTHFD2 and ALDH1L2, play critical roles in cancer survival and proliferation presumably though purine production, and are thus suggested as potential diagnostic and therapeutic targets in gastrointestinal cancer cells. THF, tetrahydrofolic acid; me-THF, N5N10-methylene-tetrahydrofolic acid; m-THF, N5-methyl-tetrahydrofolic acid; F-THF, N10-formyltetrahydrofolic acid; MET, methionine; SAM, S-adenosylmethionine; SAH, S-adenosyhomocysteine; hCYS, homocysteine; DMG, dimethylglycine; ROS, reactive oxygen species.

targets for treatment of colorectal cancer (6). The genomic analysis of clinical samples is an entry point for developments in Precision Medicine. Here we highlight recent developments in $\mathrm{C} 1$ metabolism research.

\section{Therapeutic targets in $\mathrm{C} 1$ metabolism}

Naturally, researchers have considered folate metabolism as a plausible target for disease control. Antagonism of folate metabolism has been the principal plank of chemotherapeutic concept for more than 60 years. Farber and colleagues (7) noted that folic acid could stimulate proliferation of acute lymphoblastic leukemia (ALL) cells and wondered whether the intermediates of chemical synthesis could antagonize cell proliferation. They conducted a pioneering study in which they used aminopterin, one of the above-mentioned intermediates, to induce clinical remission in patients with ALL (8). Thereafter, multiple pathways downstream of $\mathrm{C} 1$ metabolism were identified and targeted by various cytotoxic chemotherapeutic agents. For example, methotrexate (MTX), an anti-folate agent that targets dihydrofolate reductase, is used to treat various cancer and is an effective therapy for rheumatoid arthritis (RA), despite its associated toxicity (9). The first documented use of 5-fluorouracil (5-FU) was reported by Spears et al (10); it was later approved for the treatment of colorectal cancer. 5-FU is an analogue of the DNA base, uracil, and is a potent thymidine synthase inhibitor that blocks methylation of dUMP to dTMP and disrupts the folate cycle (11). Similarly, gemcitabine, another nucleotide metabolism inhibitor in the $\mathrm{C} 1$ metabolic pathway, is used to treat pancreatic cancer (12). A previous study of gemcitabine-resistant pancreatic cancer cells indicated that microRNA-1246, which belongs to a class of non-coding RNAs, is involved in the modulation of chemotherapy resistance and cancer stem cell properties, which suggests a critical role of nucleotide metabolism in cancer cell metabolism (12). The conceptual basis of 5-FU has been used to develop a thymidine analog, trifluorothymidine (TFT), as discussed below.

Recently, C1 metabolic enzymes were shown to be novel therapeutic targets for cancer. Pandey et al (13) showed that inhibition of SHMT1 with targeted siRNAs reduced tumor size in a mouse xenograft model. Pickman et al (14) demonstrated inhibition of acute myeloid leukemia cells by MTHFD2 knockdown-induced suppression of TCA in vivo. Small compounds for inhibition of SHMT1 or MTHFD2 have already been identified (15-18). These compounds may undergo further development as novel drugs for cancer therapy in the foreseeable future.

Regarding nucleotide medicine, microRNAs have been shown to exert various effects on cells, such as epigenetic reprogramming via modulation of the methylation pathway $(19,20)$. Later studies indicated that specific microRNAs, such as microRNA-302, could induce reprogramming in cancer cells, thus, identifying these as candidate moieties for treatment of refractory cancer cells from a nucleotide medicine perspective (21-23). Furthermore, microRNA-369 was shown to modulate the activity of a splicing factor of pyruvate kinase (PK), which induces metabolic reprogramming (24). Taken together, nucleotide metabolism plays a critical role in $\mathrm{C} 1$ metabolism and allows the generation of useful tools for mechanistic studies and therapeutic tools with which to target cancer cells.

Control of methylation events might be plausible, given the significance of epigenetic events with regard to the malignant phenotype of cancer $(25,26)$. Previous research has shown that a temporarily distinct subpopulation of slow-cycling melanoma 
cells in which the H3K4 demethylase JARID1B (KDM5B/ PLU-1/RBP2-H1) play a role is required for continuous tumor growth (27). These slow-cycling cells, which exhibit slow DNA replication and are likely resistant to chemotherapeutic reagents (e.g., genotoxic agents) and radiation, may be instrumental in tumor relapse and metastasis. In solid cancers, KDM family members are implicated in carcinogenesis, and knockdown of associated genes has been shown to inhibit tumorigenicity and elicit cellular senescence $(28,29)$. Several reagents, such as dimethyl sulfoxide (DMFO), have been developed to target methylation donors, ornithine decarboxylation (ODC), and polyamine metabolism and have been evaluated in clinical trials (30).

\section{Application of nucleotide analogues in C1 metabolism}

Nucleoside analogues, including deoxyadenosine analogues, adenosine analogues (31), deoxycytidine analogues, guanosine and deoxyguanosine analogues, thymidine and deoxythymidine analogues, and deoxyuridine analogues, can be used to target hepatitis B or C virus (HBV and $\mathrm{HCV}$ ), herpes simplex virus (HSV) and human immunodeficiency virus (HIV). The uracil analogue, 5-FU, contains a fluorine atom in place of hydrogen at the C-5 position (32). 5-FU is the cornerstone of treatment for various malignancies, including colon, gastric and pancreatic cancers. Current strategy for cancer treatment usually includes a combination of cytotoxic drugs and more targeted drugs that affect, for example, signal transduction pathways. Furthermore, the efficacy of the combination drug tegafur/gimeracil/oteracil (TS-1 in Japan) in patients with advanced gastric cancer has been reported in an adjuvant setting (33). Gimeracil has been reported to inhibit tegafur degradation, thus, increasing the effect of tegafur. More recently, the thymidine analog TFT has been shown to be a potent inhibitor of DNA replication. Originally, the effects of TFT were evaluated in tumors transplanted into mice in the 1960s (34). However, the short half-life of TFT, which limits its clinical use as a chemotherapeutic agent, is yet to be overcome. TFT is an antiviral drug that interferes with DNA replication. This agent is thought to overcome signaling pathways involved in resistance to 5-FU derivatives (S-1) in several model settings. 5-chloro-6-(2-iminopyrrolidin-1-yl) methyl-2,4(1H,3H)-pyrimidinedione hydrochloride (TPI) is a potent inhibitor of thymidine phosphorylase, the enzyme that degrades FTD, and thereby potentiates the efficacy of TF in vivo. A TFT:TPI molecular ratio of $2: 1$ was used in TAS-102. Evaluation of this combination demonstrated that the cytotoxicity of TFT is enhanced by TPI. Furthermore, TPI also possesses antiangiogenic properties; specifically, this agent inhibits thymidine phosphorylase (TP). Evaluation of these drugs in combination with other cytotoxic agents for treatment of various cancers has also yielded consistent results. The combinatorial use of these agents with other targeted agents synergistically downregulates signal transduction pathways responsible for tumor growth, progression and metastasis. In patients with refractory colorectal cancer, TAS-102 was associated with a significant improvement in overall survival relative to the placebo in both phase II and phase III trials $(35,36)$. Further studies to assess the efficacy of S-1 or TAS-102 in a neoadjuvant setting are underway (37-39).
The above-described results clearly demonstrate that in the future, these agents will alter the effectiveness of anti-metabolite agents used for cancer chemotherapy.

\section{Polyamines in $\mathrm{C} 1$ metabolism}

The methionine cycle produces S-adenocyl methionine (SAM), which acts as a methyl donor in methylation reactions (40). SAM is involved in the methylation of histones, DNA and RNA, as well as of lysine and arginine in general proteins. SAM is coupled with ornithine metabolic pathway. In a study of PK, which catalyzes the last step of glycolysis, PKM2 knock-down in the allele contributed to the generation of SAM in mice (24), which suggests an important role of PKM2 in the modulation of cancer phenotypes via SAM-mediated control of methylation. PKM2, which results from alternative splicing of the PK gene, was preferentially expressed in tumors relative to PKM1, which is expressed in differentiated cells. PK contributes to the production and transportation of pyruvate in the mitochondria and is thus, associated with folate production in $\mathrm{C} 1$ metabolism. This gateway function of $\mathrm{PK}$ is altered in colorectal cancer, wherein the translocation of PKM2 protein into the nucleus via TGF- $\beta$ stimulation has been observed in metastatic cancer cells (41); notably, pyruvate dehydrogenase is also affected in cancer cells (42).

SAM production is associated with polyamine metabolism in which ornithine decarboxylation (ODC) functions as a restricting step in the metabolic flow (43). Studies of an ODC enzyme revealed the characteristic cancer stem cell properties of fluorescent cancer cells harboring a GFP-ODC enzyme fusion cassette (44-46). These GFP-ODC labeled cancer cells exhibited the most aggressive tumorigenicity in immunodeficient mice, were resistant to chemotherapy and radiation therapy and exhibited reduced production of reactive oxygen species (ROS). A trans-omics mathematical analysis that linked metabolome data with transcriptome data revealed novel functions of the ornithine metabolic pathway in cancer stem cells (47). Given that ornithine is located upstream of polyamine metabolism, the polyamine flow might play a role in the maintenance of cancer stemness. Thus, $\mathrm{C} 1$ metabolism helps to control treatment-refractory cancer stem cells.

\section{Diadenosine phosphate hydrolases in $\mathrm{C} 1$ metabolism}

Although genetic alterations are not the sole pathogenetic mechanism of carcinogenesis, these factors undoubtedly play a significant role in disease initiation and progression (48-50). Studies of hereditary diseases that are known to predispose to cancer have indicated the involvement of ectopically activated oncogenes and the inhibition of tumor suppressor genes (51). In the 1990s, numerous studies suggested that in cancer patients, commonly deleted genomic regions might contain tumor suppressor genes (52); accordingly, introduction of these missing genes to cancer cells might inactivate tumor cell proliferation and cell cycle progression and thus suppress tumorigenicity (53). Positional cloning approaches to the identification of critical genes in the common fragile sites on chromosome 3p14 led to the identification of the fragile histidine triad (FHIT) gene, which encodes an enzyme with dinucleotide hydrolase activity (diadenosine tri-phosphate 


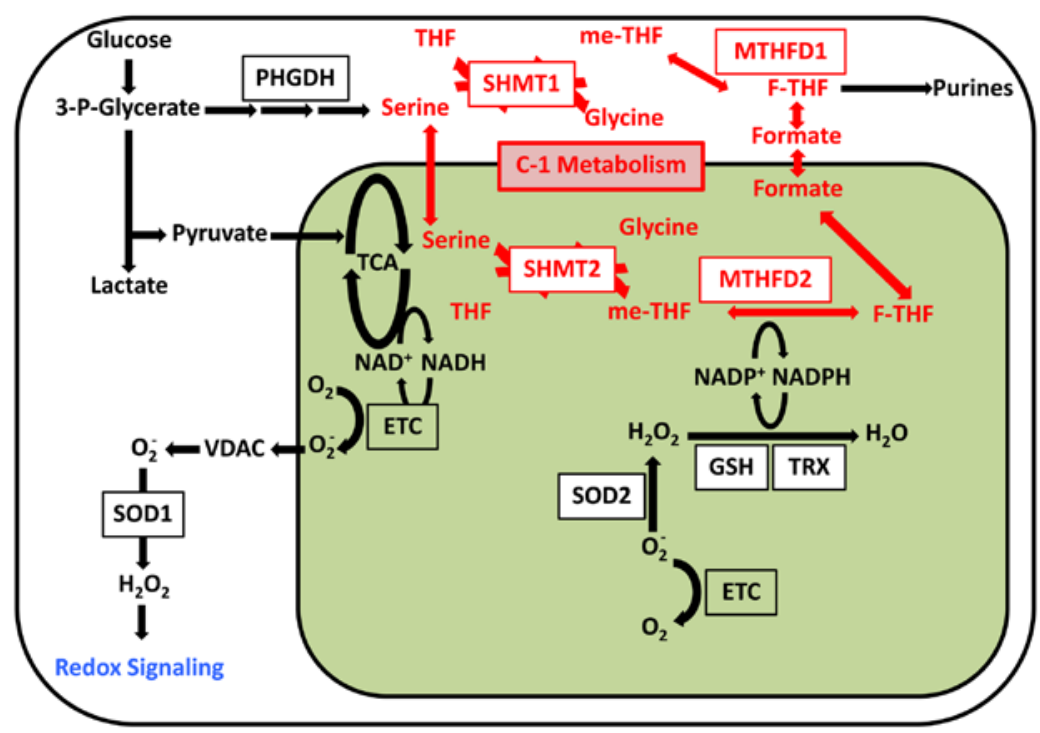

Figure 2. One-carbon metabolism in mitochondria. One-carbon metabolism comprises three critical reactions: the folate and methionine cycles and the transsulfuration pathway. In the folate cycle, glycine and serine fuel mitochondrial enzymes via purine production. In the methionine cycle, S-adenocyl methionine (SAM) serves both hydrocarbons and polyamines. The trans-sulfuration pathway is critical for the synthesis of glutathione, which is involved in the production of reactive oxygen species. Acting in unison, these molecules promote the survival and maintenance of gastrointestinal cancer cells. NAD, nicotinamide adenine dinucleotide; NADH, reduced nicotinamide adenine dinucleotide; ETC, electron transport chain; GSH, glutathione; PHGDH, 3-phosphoglycerate dehydrogenase; SOD1, superoxide dismutase 1; SOD1, superoxide dismutase 2; TRX, thioredoxin; VDAC, voltage dependent anion channel 1.

hydrolase) and a role in purine metabolism (54). A subsequent biochemical study indicated the importance of His96 as a catalyst for the hydrolysis of phosphoanhydrides such as Ap3A (55). More than 50\% of human tumors exhibit focal deletion of this gene (56). Experiments in mice have indicated a deficiency in FHIT-induced genomic instability and spontaneous tumor formation, both of which were suppressed by the introduction of FHIT $(3,57)$.

Studies of the FHIT loci genomic structure identified LINE-1, a human transposable element that is presumably involved in genomic deletion breakpoints associated with cancer $(58,59)$. Since aphidicholine, an inhibitor of DNA polymerase $\alpha$ and $\delta$, is known to affect the fragility of the above-mentioned common fragile sites (56), fragility in cancer cells might involve processes such as replication, recombination and DNA repair. Indeed, studies of gene function have indicated the involvement of Fhit protein in checkpoint system activation in response to genomic damage (60). In cancer, alterations to this checkpoint response have been linked to the activation of an Akt-survivin pathway-mediated cell survival mechanism (61). The mechanism by which the above-mentioned phenomenon occurs in tumors remains to be elucidated; however, DNA repair presumably requires the repair enzymes to appropriately incorporate nucleotide bases into DNA (3). Therefore, this historically important discovery of FHIT from the most active common fragile sites in the human genome indicates the homology of the encoded protein with dinucleotide hydrolase (62) and suggests that $\mathrm{C} 1$ metabolism leads to nucleotide metabolism in cancer cells.

\section{ROS in C1 metabolism}

Mitochondrial quality is known to influence cellular differentiation. For example, certain mutations in mitochondrial DNA (mtDNA) affect cellular reprogramming. Reprogramming induction in fibroblasts harboring mtDNA mutations revealed drastically reduced reprogramming efficiency of these cells relative to that of wild-type fibroblast cells (63). Reduced reprogramming efficiency has also been observed in human cells that harbor large mtDNA deletions (64), as well as in clonal human fibroblast cells with very high frequency of mt-tRNA point mutations. In addition, mtDNA has been suggested to affect reprogramming efficiency $(57,58)$. However, the induced pluripotent stem cell lines showed different pathological mtDNA point mutations (20,25,63-66). In these cells, no significant difference in reprogramming efficiency was observed between the normal and mutated lines. Many studies have associated heteroplasmic mtDNA mutations with specific segregation patterns during reprogramming. This phenomenon was not only observed in the induced pluripotent stem cells, but also in mouse germ cells and during epiblast differentiation in monkey embryos $(11,67)$.

Furthermore, tDNA mutation was found to induce ROS. ROS signaling determines cell fate. For example, mitochondrial ROS was shown to induce differentiation of hematopoietic stem cells (HSCs) (9,30). Therefore, ROS was thought to mediate signaling and thus affect cell differentiation. Induced pluripotent stem cells with mtDNA mutations retain high levels of ROS (63), although this phenotype can be rescued via treatment with antioxidants such as n-acetyl-1cysteine (NAC). Altered ROS signaling is thought to induce the mtDNA mutation phenotype in stem cells (63). Therefore, the mitochondria is an organelle involved in signal transduction (Fig. 2).

\section{Roles in cancer stem cell control}

ROS such as superoxide $\left(\mathrm{O}_{2}^{-}\right)$, hydrogen peroxide $\left(\mathrm{H}_{2} \mathrm{O}_{2}\right)$, and hydroxyl radical $(\mathrm{OH})$, are highly chemically reactive species derived from molecular oxygen $(68,69)$. ROS are generated 
in the mitochondria (69). ROS can also be produced by various oxidases (e.g., NADPH oxidases and peroxidases) in different cellular compartments or organelles, such as the cell membranes, peroxisomes and the endoplasmic reticulum (70). Furthermore, chemotherapy, radioactivity, and even smoking can increase cellular ROS levels $(66,71,72)$. A low level of ROS promotes cell proliferation and growth and increases cell survival (73). In contrast, a high level of ROS can cause cellular toxicity and trigger apoptosis (74,75). Cellular antioxidant systems can scavenge ROS and prevent irreversible cellular oxidative damage (76). It is important for cells to balance ROS generation and antioxidant activity, and redox regulation of cellular processes is essential for growth and development. ROS levels are increased in many cancer cells, and this is in part due to the higher metabolism rate $(65,77)$. Aberrant ROS levels can elicit cancer cell apoptosis and necrosis (78). Cancer cells have a high antioxidant capacity to counteract and scavenge ROS. Because this high antioxidant capacity enhances cell survival and impairs cellular responses to anticancer therapy (79), induction of ROS-mediated damage in cancer cells with use of appropriate pharmacological agents that either promote ROS generation beyond the cellular antioxidative capacity or disable the cellular antioxidant system, has been considered as a radical therapeutic strategy for preferential targeting of cancer cells (79). Recently, cancer stem cells (CSCs) have gained attention as a subpopulation of cancer cells with stem cell-like properties and characteristics; these cells have been identified in the context of various cancers, including leukemia (80), breast (64) and pancreatic cancer (81). CSCs have the capacity to self-renew and differentiate and are thought to be responsible for cancer recurrence after chemotherapy or radiotherapy because of their ability to survive treatment and quickly generate new tumors $(82,83)$. Characterization of CSCs have led to a perspective in which cancer therapeutic strategies should target not only normal cancer cells, but also CSCs. Given the importance of redox balance in cancer cells, conventional therapies (chemotherapy or radiotherapy) that target the redox balance could kill most cancer cells $(67,79,84)$. However, the unique redox balance in CSCs and the underlying mechanisms that protect CSCs from ROS-mediated cell killing have not been fully elucidated $(63,85,86)$.

\section{Acknowledgements}

The present review was supported in part by a Grant-in-Aid for Scientific Research from the Ministry of Education, Culture, Sports, Science and Technology; a Grant-in-Aid from the Third Comprehensive 10-year Strategy for Cancer Control, Ministry of Health, Labor and Welfare; a grant from the Kobayashi Cancer Research Foundation; a grant from the Princess Takamatsu Cancer Research Fund, Japan; a grant from the National Institute of Biomedical Innovation; and a grant from the Osaka University Drug Discovery Funds. A.H. is a research fellow of the Japan Society for the Promotion of Science. Partial support was received from Taiho Pharmaceutical, Co., Ltd., (H.I., J.K. and M.M.), Chugai, Co., Ltd., Yakult Honsha, Co., Ltd., Merck, Co., Ltd., Takeda Science Foundation and Takeda Medical Research Foundation (M.K., M.M., N.N. and H.I.) through institutional endowments.

\section{References}

1. Ghosh D and Poisson LM: 'Omics' data and levels of evidence for biomarker discovery. Genomics 93: 13-16, 2009.

2. Zong WX, Rabinowitz JD and White E: Mitochondria and Cancer. Mol Cell 61: 667-676, 2016.

3. Locasale JW: Serine, glycine and one-carbon units: Cancer metabolism in full circle. Nat Rev Cancer 13: 572-583, 2013.

4. Hanley MP and Rosenberg DW: One-carbon metabolism and colorectal cancer: Potential mechanisms of chemoprevention. Curr Pharmacol Rep 1: 197-205, 2015.

5. Padmanabhan $\mathrm{N}$ and Watson ED: Lessons from the one-carbon metabolism: Passing it along to the next generation. Reprod Biomed Online 27: 637-643, 2013.

6. Miyo M, Konno M, Colvin H, Nishida N, Koseki J, Kawamoto K, Tsunekuni K, Nishimura J, Hata T, Takemasa I, et al: The importance of mitochondrial folate enzymes in human colorectal cancer. Oncol Rep 37: 417-425, 2016.

7. Farber S, Cutler EC, Hawkins JW, Harrison JH, Peirce EC II and Lenz GG: The action of pteroylglutamic conjugates on man. Science 106: 619-621, 1947.

8. Farber S, Diamond LK, Mercer RD, Sylvester RF Jr and Wolff JA: Temporary remissions in acute leukemia in children produced by folic acid antagonist, 4-aminopteroyl-glutamic acid. N Engl J Med 238: 787-793, 1948.

9. Chabner BA and Roberts TG Jr: Timeline: Chemotherapy and the war on cancer. Nat Rev Cancer 5: 65-72, 2005.

10. Spears CP, Shahinian AH, Moran RG, Heidelberger C and Corbett TH: In vivo kinetics of thymidylate synthetase inhibition of 5-fluorouracil-sensitive and -resistant murine colon adenocarcinomas. Cancer Res 42: 450-456, 1982.

11. Burris HA III, Moore MJ, Andersen J, Green MR, Rothenberg ML, Modiano MR, Cripps MC, Portenoy RK, Storniolo AM, Tarassoff $\mathrm{P}$, et al: Improvements in survival and clinical benefit with gemcitabine as first-line therapy for patients with advanced pancreas cancer: A randomized trial. J Clin Oncol 15: 2403-2413, 1997.

12. Hasegawa S, Eguchi H, Nagano H, Konno M, Tomimaru Y, Wada H, Hama N, Kawamoto K, Kobayashi S, Nishida N, et al: MicroRNA-1246 expression associated with CCNG2-mediated chemoresistance and stemness in pancreatic cancer. Br J Cancer 111: 1572-1580, 2014.

13. Pandey S, Garg P, Lee S, Choung HW, Choung YH, Choung PH and Chung JH: Nucleotide biosynthesis arrest by silencing SHMT1 function via vitamin B6-coupled vector and effects on tumor growth inhibition. Biomaterials 35: 9332-9342, 2014.

14. Pikman Y, Puissant A, Alexe G, Furman A, Chen LM, Frumm SM, Ross L, Fenouille N, Bassil CF, Lewis CA, et al: Targeting MTHFD2 in acute myeloid leukemia. J Med Chem 213: 1285-1306, 2016

15. Marani M, Paone A, Fiascarelli A, Macone A, Gargano M, Rinaldo S, Giardina G, Pontecorvi V, Koes D, McDermott L, et al: A pyrazolopyran derivative preferentially inhibits the activity of human cytosolic serine hydroxymethyltransferase and induces cell death in lung cancer cells. Oncotarget 7: 4570-4583, 2016.

16. Paiardini A, Fiascarelli A, Rinaldo S, Daidone F, Giardina G, Koes DR, Parroni A, Montini G, Marani M, Paone A, et al: Screening and in vitro testing of antifolate inhibitors of human cytosolic serine hydroxymethyltransferase. ChemMedChem 10: 490-497, 2015.

17. Witschel MC, Rottmann M, Schwab A, Leartsakulpanich U, Chitnumsub P, Seet M, Tonazzi S, Schwertz G, Stelzer F, Mietzner T, et al: Inhibitors of plasmodial serine hydroxymethyltransferase (SHMT): Cocrystal structures of pyrazolopyrans with potent blood- and liver-stage activities. J Med Chem 58: 3117-3130, 2015.

18. Gustafsson R, Jemth AS, Gustafsson Sheppard N, Färnegårdh K, Loseva O, Wiita E, Bonagas N, Dahllund L, Llona-Minguez S and Häggblad M: Crystal structure of the emerging cancer target MTHFD2 in complex with a substrate-based inhibitor. Cancer Res: Nov 29, 2016 (Epub ahead of print).

19. Miyoshi N, Ishii H, Nagano H, Haraguchi N, Dewi DL, Kano Y, Nishikawa S, Tanemura M, Mimori K, Tanaka F, et al: Reprogramming of mouse and human cells to pluripotency using mature microRNAs. Cell Stem Cell 8: 633-638, 2011.

20. Anokye-Danso F, Trivedi CM, Juhr D, Gupta M, Cui Z, Tian Y, Zhang Y, Yang W, Gruber PJ, Epstein JA, et al: Highly efficient miRNA-mediated reprogramming of mouse and human somatic cells to pluripotency. Cell Stem Cell 8: 376-388, 2011. 
21. Miyoshi N, Ishii H, Nagai K, Hoshino H, Mimori K, Tanaka F, Nagano H, Sekimoto M, Doki Y and Mori M: Defined factors induce reprogramming of gastrointestinal cancer cells. Proc Natl Acad Sci USA 107: 40-45, 2010.

22. Dewi D, Ishii H, Haraguchi N, Nishikawa S, Kano Y, Fukusumi T, Ohta K, Miyazaki S, Ozaki M, Sakai D, et al: Reprogramming of gastrointestinal cancer cells. Cancer Sci 103: 393-399, 2012.

23. Ogawa H, Wu X, Kawamoto K, Nishida N, Konno M, Koseki J, Matsui H, Noguchi K, Gotoh N, Yamamoto T, et al: MicroRNAs induce epigenetic reprogramming and suppress malignant phenotypes of human colon cancer cells. PLoS One 10: e0127119, 2015.

24. Konno M, Koseki J, Kawamoto K, Nishida N, Matsui H, Dewi DL, Ozaki M, Noguchi Y, Mimori K, Gotoh N, et al: Embryonic microRNA-369 controls metabolic splicing factors and urges cellular reprograming. PLoS One 10: e0132789, 2015.

25. Avgustinova A and Benitah SA: The epigenetics of tumour initiation: Cancer stem cells and their chromatin. Curr Opin Genet Dev 36: 8-15, 2016.

26. Rotili D and Mai A: Targeting histone demethylases: A new avenue for the fight against cancer. Genes Cancer 2: 663-679, 2011.

27. Roesch A, Fukunaga-Kalabis M, Schmidt EC, Zabierowski SE, Brafford PA, Vultur A, Basu D, Gimotty P, Vogt T and Herlyn M: A temporarily distinct subpopulation of slow-cycling melanoma cells is required for continuous tumor growth. Cell 141: 583-594, 2010.

28. Kano Y, Konno M, Ohta K, Haraguchi N, Nishikawa S, Kagawa Y, Hamabe A, Hasegawa S, Ogawa H, Fukusumi T, et al: Jumonji/Arid1b (Jarid1b) protein modulates human esophageal cancer cell growth. Mol Clin Oncol 1: 753-757, 2013.

29. Ohta K, Haraguchi N, Kano Y, Kagawa Y, Konno M, Nishikawa S, Hamabe A, Hasegawa S, Ogawa H, Fukusumi T, et al: Depletion of JARID1B induces cellular senescence in human colorectal cancer. Int J Oncol 42: 1212-1218, 2013.

30. Casero RAJ Jr and Marton LJ: Targeting polyamine metabolism and function in cancer and other hyperproliferative diseases. Nat Rev Drug Discov 6: 373-390, 2007.

31. Warren TK, Jordan R, Lo MK, Ray AS, Mackman RL, Soloveva V, Siegel D, Perron M, Bannister R, Hui HC, et al: Therapeutic efficacy of the small molecule GS-5734 against Ebola virus in rhesus monkeys. Nature 531: 381-385, 2016.

32. Longley DB, Harkin DP and Johnston PG: 5-fluorouracil: Mechanisms of action and clinical strategies. Nat Rev Cancer 3 : 330-338, 2003.

33. Sakuramoto S, Sasako M, Yamaguchi T, Kinoshita T, Fujii M, Nashimoto A, Furukawa H, Nakajima T, Ohashi Y, Imamura H, et al; ACTS-GC Group: Adjuvant chemotherapy for gastric cancer with S-1, an oral fluoropyrimidine. N Engl J Med 357: $1810-1820,2007$

34. Kaufman HE and Heidelberger C: Therapeutic antiviral Action of 5-trifluoromethyl-2'-deoxyuridine in Herpes simplex keratitis Science 145: 585-586, 1964.

35. Mayer RJ, Van Cutsem E, Falcone A, Yoshino T, GarciaCarbonero R, Mizunuma N, Yamazaki K, Shimada Y, Tabernero J, Komatsu Y, et al; RECOURSE Study Group: Randomized trial of TAS-102 for refractory metastatic colorectal cancer. N Engl J Med 372: 1909-1919, 2015

36. Yoshino T, Mizunuma N, Yamazaki K, Nishina T, Komatsu Y, Baba H, Tsuji A, Yamaguchi K, Muro K, Sugimoto N, et al: TAS-102 monotherapy for pretreated metastatic colorectal cancer: A double-blind, randomised, placebo-controlled phase 2 trial. Lancet Oncol 13: 993-1001, 2012.

37. Honma Y, Yamada Y, Terazawa T, Takashima A, Iwasa S, Kato K, Hamaguchi T, Shimada Y, Ohashi M, Morita S, et al: Feasibility of neoadjuvant S-1 and oxaliplatin followed by surgery for resectable advanced gastric adenocarcinoma. Surg Today 46: 1076-1082, 2016.

38. Uehara $\mathrm{K}$ and Nagino $\mathrm{M}$ : Neoadjuvant treatment for locally advanced rectal cancer: A systematic review. Surg Today 46 : 161-168, 2016.

39. Park IJ, Kim JY, Yu CS, Lee JS, Lim SB, Lee JL, Yoon YS, Kim CW and Kim JC: Preoperative chemoradiotherapy for clinically diagnosed T3N0 rectal cancer. Surg Today 46: 90-96, 2016.

40. Su X, Wellen KE and Rabinowitz JD: Metabolic control of methylation and acetylation. Curr Opin Chem Biol 30: 52-60, 2016.
41. Hamabe A, Konno M, Tanuma N, Shima H, Tsunekuni K, Kawamoto K, Nishida N, Koseki J, Mimori K, Gotoh N, et al: Role of pyruvate kinase $\mathrm{M} 2$ in transcriptional regulation leading to epithelial-mesenchymal transition. Proc Natl Acad Sci USA 111: 15526-15531, 2014

42. Hamabe A, Yamamoto H, Konno M, Uemura M, Nishimura J, Hata T, Takemasa I, Mizushima T, Nishida N, Kawamoto K, et al: Combined evaluation of hexokinase 2 and phosphorylated pyruvate dehydrogenase-E1 $\alpha$ in invasive front lesions of colorectal tumors predicts cancer metabolism and patient prognosis. Cancer Sci 105: 1100-1108, 2014.

43. Gerner EW and Meyskens FL Jr: Polyamines and cancer: Old molecules, new understanding. Nat Rev Cancer 4: 781-792, 2004.

44. Hayashi K, Tamari K, Ishii H, Konno M, Nishida N, Kawamoto K, Koseki J, Fukusumi T, Kano Y, Nishikawa S, et al: Visualization and characterization of cancer stem-like cells in cervical cancer. Int J Oncol 45: 2468-2474, 2014.

45. Kano Y, Konno M, Kawamoto K, Tamari K, Hayashi K, Fukusumi T, Satoh T, Tanaka S, Ogawa K, Mori M, et al: Novel drug discovery system for cancer stem cells in human squamous cell carcinoma of the esophagus. Oncol Rep 31: 1133-1138, 2014.

46. Tamari K, Hayashi K, Ishii H, Kano Y, Konno M, Kawamoto K, Nishida N, Koseki J, Fukusumi T, Hasegawa S, et al: Identification of chemoradiation-resistant osteosarcoma stem cells using an imaging system for proteasome activity. Int J Oncol 45: 2349-2354, 2014

47. Koseki J, Matsui H, Konno M, Nishida N, Kawamoto K, Kano Y, Mori M, Doki Y and Ishii H: A Trans-omics mathematical analysis reveals novel functions of the ornithine metabolic pathway in cancer Stem cells. Sci Rep 6: 20726, 2016.

48. Hanahan D and Weinberg RA: The hallmarks of cancer. Cell 100: 57-70, 2000.

49. Hanahan D and Weinberg RA: Hallmarks of cancer: The next generation. Cell 144: 646-674, 2011.

50. Nowell PC: Foundations in cancer research. Chromosomes and cancer: The evolution of an idea. Adv Cancer Res 62: 1-17, 1993.

51. Nowell PC and Croce CM: Chromosomes, genes, and cancer. Am J Pathol 125: 7-15, 1986.

52. Weinberg RA: Tumor suppressor genes. Science 254: 1138-1146, 1991.

53. Sherr CJ: Cancer cell cycles. Science 274: 1672-1677, 1996.

54. Ohta M, Inoue H, Cotticelli MG, Kastury K, Baffa R, Palazzo J, Siprashvili Z, Mori M, McCue P, Druck T, et al: The FHIT gene, spanning the chromosome 3p14.2 fragile site and renal carcinoma-associated $\mathrm{t}(3 ; 8)$ breakpoint, is abnormal in digestive tract cancers. Cell 84: 587-597, 1996.

55. Huang K and Frey PA: Engineering human Fhit, a diadenosine triphosphate hydrolase, into an efficient dinucleoside polyphosphate synthase. J Am Chem Soc 126: 9548-9549, 2004.

56. Huebner $\mathrm{K}$ and Croce CM: FRA3B and other common fragile sites: The weakest links. Nat Rev Cancer 1: 214-221, 2001.

57. Dumon KR, Ishii H, Fong LY, Zanesi N, Fidanza V, Mancini R, Vecchione A, Baffa R, Trapasso F, During MJ, et al: FHIT gene therapy prevents tumor development in Fhit-deficient mice. Proc Natl Acad Sci USA 98: 3346-3351, 2001.

58. Inoue H, Ishii H, Alder H, Snyder E, Druck T, Huebner K and Croce CM: Sequence of the FRA3B common fragile region: Implications for the mechanism of FHIT deletion. Proc Natl Acad Sci USA 94: 14584-14589, 1997.

59. Mimori K, Druck T, Inoue H, Alder H, Berk L, Mori M, Huebner K and Croce CM: Cancer-specific chromosome alterations in the constitutive fragile region FRA3B. Proc Natl Acad Sci USA 96: 7456-7461, 1999.

60. Ishii H, Mimori K, Inoue H, Inageta $T$, Ishikawa K, Semba S, Druck T, Trapasso F, Tani K, Vecchione A, et al: Fhit modulates the DNA damage checkpoint response. Cancer Res 66: $11287-11292,2006$.

61. Semba S, Trapasso F, Fabbri M, McCorkell KA, Volinia S, Druck T, Iliopoulos D, Pekarsky Y, Ishii H, Garrison PN, et al: Fhit modulation of the Akt-survivin pathway in lung cancer cells: Fhit-tyrosine 114 (Y114) is essential. Oncogene 25: 2860-2872, 2006.

62. Arlt MF, Casper AM and Glover TW: Common fragile sites. Cytogenet Genome Res 100: 92-100, 2003.

63. Dayem AA, Choi HY, Kim JH and Cho SG: Role of oxidative stress in stem, cancer, and cancer stem cells. Cancers (Basel) 2: 859-884, 2010.

64. Al-Hajj M, Wicha MS, Benito-Hernandez A, Morrison SJ and Clarke MF: Prospective identification of tumorigenic breast cancer cells. Proc Natl Acad Sci USA 100: 3983-3988, 2003. 
65. Ambrosone CB: Oxidants and antioxidants in breast cancer Antioxid Redox Signal 2: 903-917, 2000.

66. Barreiro E, Peinado VI, Galdiz JB, Ferrer E, Marin-Corral J, Sánchez F, Gea J and Barberà JA; ENIGMA in COPD Project: Cigarette smoke-induced oxidative stress: A role in chronic obstructive pulmonary disease skeletal muscle dysfunction. Am J Respir Crit Care Med 182: 477-488, 2010.

67. Cairns RA, Harris IS and Mak TW: Regulation of cancer cell metabolism. Nat Rev Cancer 11: 85-95, 2011.

68. Kobayashi CI and Suda T: Regulation of reactive oxygen species in stem cells and cancer stem cells. J Cell Physiol 227: 421-430, 2012.

69. Turrens JF: Mitochondrial formation of reactive oxygen species. J Physiol 552: 335-344, 2003.

70. Dickinson BC and Chang CJ: Chemistry and biology of reactive oxygen species in signaling or stress responses. Nat Chem Biol 7: 504-511, 2011

71. Lee KW, Lee DJ, Lee JY, Kang DH, Kwon J and Kang SW: Peroxiredoxin II restrains DNA damage-induced death in cancer cells by positively regulating JNK-dependent DNA repair. J Biol Chem 286: 8394-8404, 2011.

72. Phillips TM, McBride WH and Pajonk F: The response of CD24 low/CD $44^{+}$breast cancer-initiating cells to radiation. J Natl Cancer Inst 98: 1777-1785, 2006

73. Giannoni E, Buricchi F, Raugei G, Ramponi G and Chiarugi P Intracellular reactive oxygen species activate $\mathrm{Src}$ tyrosine kinase during cell adhesion and anchorage-dependent cell growth. Mol Cell Biol 25: 6391-6403, 2005.

74. Hoeijmakers JH: DNA damage, aging, and cancer. N Engl J Med 361: 1475-1485, 2009.

75. Yee $\mathrm{C}$, Yang $\mathrm{W}$ and Hekimi S: The intrinsic apoptosis pathway mediates the pro-longevity response to mitochondrial ROS in C. elegans. Cell 157: 897-909, 2014.
76. Fruehauf JP and Meyskens FL Jr: Reactive oxygen species: A breath of life or death? Clin Cancer Res 13: 789-794, 2007.

77. Szatrowski TP and Nathan CF: Production of large amount of hydrogen peroxide by human tumor cells. Cancer Res 51: 794-798, 1991 .

78. Halliwell B: Oxidative stress and cancer: Have we moved forward? Biochem J 401: 1-11, 2007.

79. Trachootham D, Alexandre J and Huang P: Targeting cancer cells by ROS-mediated mechanisms: A radical therapeutic approach? Nat Rev Drug Discov 8: 579-591, 2009.

80. Chan SM and Majeti R: Role of DNMT3A, TET2, and IDH1/2 mutations in pre-leukemic stem cells in acute myeloid leukemia. Int J Hematol 98: 648-657, 2013.

81. Hermann PC, Huber SL, Herrler T, Aicher A, Ellwart JW, Guba M, Bruns CJ and Heeschen C: Distinct populations of cancer stem cells determine tumor growth and metastatic activity in human pancreatic cancer. Cell Stem Cell 1: 313-323, 2007.

82. Eyler CE and Rich JN: Survival of the fittest: Cancer stem cells in therapeutic resistance and angiogenesis. J Clin Oncol 26: 2839-2845, 2008.

83. Kurtova AV, Xiao J, Mo Q, Pazhanisamy S, Krasnow R, Lerner SP, Chen F, Roh TT, Lay E, Ho PL, et al: Blocking PGE -induced $_{2}$ tumour repopulation abrogates bladder cancer chemoresistance. Nature 517: 209-213, 2015.

84. Schafer ZT, Grassian AR, Song L, Jiang Z, Gerhart-Hines Z, Irie HY, Gao S, Puigserver P and Brugge JS: Antioxidant and oncogene rescue of metabolic defects caused by loss of matrix attachment. Nature 461: 109-113, 2009.

85. Wang K, Zhang T, Dong Q, Nice EC, Huang C and Wei Y: Redox homeostasis: The linchpin in stem cell self-renewal and differentiation. Cell Death Dis 4: e537, 2013.

86. Shi X, Zhang Y, Zheng J and Pan J: Reactive oxygen species in cancer stem cells. Antioxid Redox Signal 16: 1215-1228, 2012. 